\title{
O PRONTUÁRIO DO PACIENTE E OS PRESSUPOSTOS ARQUIVÍSTICOS: ESTREITAS E PROFÍCUAS INTERLOCUÇÕES
}

\section{REGISTROS DE PACIENTES Y LOS SUPUESTOS ARCHIVERO: LOS DIÁLOGOS ESTRECHA Y FRUCTÍFERA}

\author{
Letícia Gorri Molina - leticiamolina@uel.br \\ Doutoranda do Programa de Pós-Graduação da UNESP/Marília. \\ Docente da Universidade Estadual de Londrina. \\ Rosane Suely Alvares Lunardelli - lunardell@uel.br \\ Doutora em Estudos da Linguagem. \\ Docente da Universidade Estadual de Londrina.
}

\begin{abstract}
Resumo
Considerado documento multifuncional para a área da Saúde, o Prontuário do Paciente é abordado em seus suportes eletrônico e papel. Em que pese as dificuldades encontradas na implantação e utilização do Prontuário Eletrônico do Paciente, o cotejo entre os dois formatos acima mencionados, indica a primazia do Prontuário Eletrônico e o caracteriza como um elemento preponderante no que tange à melhoria dos sistemas e serviços de saúde de um país. Dadas as suas especificidades, os requisitos fundamentais à sua plena utilização, entre outros fatores, ressalta-se a relevância dos pressupostos arquivísticos na organização da informação e do conhecimento registrados nesses dossiês.
\end{abstract}

\section{Palavras-chave}

Organização da informação. Prontuário do paciente. Prontuário eletrônico do paciente (pep). Arquivologia. Funções arquivísticas

\section{INTRODUÇÃO}

A organização da informação e do conhecimento com vistas à sua apropriação tem sido uma das necessidades mais prementes da humanidade. Atualmente, porém, de acordo com Souza (1998, p.7), "[...] ficou ainda mais evidente o quanto é imprescindível a organização desse saber". Tal afirmação ancora-se em dois fatos que permeiam a 
sociedade pós-moderna: a quantidade e variedade de informação que circulam em diferentes suportes e o número expressivo de pessoas que buscam acessá-las.

A informação, portanto, seja ela registrada de forma escrita, oral ou audiovisual, é o elemento básico para a produção e disseminação do conhecimento. É considerada, quando percebida e assimilada, o instrumento modificador da consciência do ser humano, uma vez que acrescenta e reformula estruturas trazendo conhecimento e benefícios ao indivíduo que a possui.

Em outros termos, Choo (2003, p.59) explicita o propósito da informação ao argumentar que ela "[...] destina-se à criação e aplicação de conhecimento através de processos de interpretação e de decisão".

Dentro dessa linha de raciocínio, Nonaka e Takeuchi, (1997), esclarecem que a informação é um fluxo de mensagens, ao passo que o conhecimento só é possível mediante esse fluxo agregado de crenças e valores essencialmente humanos. Nas palavras de Wilson (2006), aquilo que se sabe pode ser definido como conhecimento, o que envolve somente processos mentais, os quais estão em constante mudança à medida que novas informações vão surgindo e sendo adquiridas.

O conhecimento, sob esse enfoque, pode ser considerado um processo de abstração pessoal ou um fluxo de informação que se potencializa, pois de acordo com Araújo (2002, p. 239), “[...] é obtido como resultante de processos mentais desencadeados a partir da informação, pela ação do sujeito, considerando-se aspectos objetivos e subjetivos".

Com o intuito de ressaltar a intervenção humana no processo de transformação da informação em conhecimento, Probst (2006) afirma que o conhecimento é, portanto, o conjunto da cognição e das habilidades utilizadas pelos seres humanos para resolverem problemas. Ele é fundamentado em dados e informações, mas, diferentemente deles, necessita competências pessoais.

Nessa perspectiva, Davenport (2000, p. 19) define conhecimento como

[...] a informação mais valiosa e, conseqüentemente, mais difícil de gerenciar. É valiosa precisamente porque alguém deu à informação um contexto, um significado, uma interpretação; alguém refletiu sobre o conhecimento acrescentou a ele sua própria sabedoria, considerou suas implicações mais amplas. 
Ressaltada a importância do conhecimento em um mundo atual, globalizado e em constante mutação, torna-se imperativa a sua socialização, pois, como afirma Mc Inerney (2006, p. 57), "compartilhar conhecimento pressupõe um interesse ativo no aprendizado, na inovação, na mudança constante e no compartilhamento daquilo que as pessoas conhecem [...]".

Durante muito tempo, acreditou-se que a disponibilização dos estoques informacionais seriam suficientes para a sua socialização. Mas atualmente entende-se que é essencial a existência de uma forma de organização que garanta o seu compartilhamento.

Consubstanciando a afirmação, Dalhberg (1993, p.214) argumenta que

vivemos em um mundo dominado pela informação, a qual necessita ser urgentemente ordenada e compendiada para transformá-la em conhecimento disponível, tanto para usos e finalidades pessoais como para a tomada de decisões públicas, em termos de aplicação política, cultural, industrial, comercial ou social (grifo nosso).

Dentre as diversas áreas do conhecimento, é foco deste estudo a área médica cuja informação é considerada elemento de sobrevivência dos profissionais "que atuam nas trincheiras da saúde, e também como garantia de melhor qualidade de vida para a população [...]" (MEDEIROS, 2002, p.119) e mais especificamente, as informações a respeito do paciente ou o prontuário do paciente ${ }^{1}$.

A elevada quantidade de prontuários, aliada à escassez de espaços físicos e a inexistência de uma metodologia sistemática de acondicionamento e recuperação desses documentos, constituem-se atualmente em imensos problemas que demandam análises e ações urgentes. Caracterizado como documento arquivístico, - uma vez que foi criado em consequência das atividades ou funções da instituição que o produziu -, destaca-se a necessidade de se observar os pressupostos arquivísticos na organização dessa informação e/ou conhecimento, por meio de processos, políticas e atividades de tratamento documentário.

Em uma perspectiva mais ampla, ao justificar a necessidade de parcerias entre os profissionais da Saúde e os da Ciência da Informação, Mota; Babêtto (.s.d.,p.1) argumentam que a Ciência da Informação possui uma abordagem das questões e

\footnotetext{
${ }^{1}$ É importante mencionar que a designação dada ao registro de informações de um determinado paciente sofreu modificação. Com o intuito de evidenciar o papel e os direitos do paciente, o Prontuário Médico atualmente é denominado Prontuário do Paciente.
} 
problemas relacionados ao fluxo da informação que pode contribuir sobremaneira para o bom andamento das atividades na área da saúde. Os autores citados, no mesmo texto e página, complementam a proposição afirmando que

a interdisciplinaridade entre estas duas áreas pode ser considerada como um grande avanço [também] para a resolução dos problemas que surgem em decorrência do não gerenciamento correto dos fluxos informacionais oriundos da inserção das tecnologias da informática no âmbito da saúde.

\section{CIÊNCIA DA INFORMAÇÃO E A ARQUIVOLOGIA}

A Ciência da Informação, em seu desenvolvimento, tem recebido influências de diferentes áreas do conhecimento, como a Linguistica, Filosofia, Biologia, Psicologia, Matemática entre outras. Assim, essas diferentes visões teóricas se refletem nas diversas definições e significações do seu objeto de estudo, a "informação".

Em meados do Século XX, a Ciência da Informação era orientada por um paradigma físico, considerando a informação como um signo físico, disseminado por sinais elétricos, "implica numa analogia entre a veiculação física de um sinal e a transmissão de uma mensagem, cujos aspectos semânticos e pragmáticos intimamente relacionados ao uso diário do termo informação são explicitamente descartados por Shannon" (CAPURRO, 2003, p.7).

Esse paradigma foi substituído pelo paradigma cognitivo, considerando a busca da informação como um processo cognitivo e de suprimento das necessidades do sujeito. "[...] trata de ver de que forma os processos informativos transformam ou não o sujeito, entendido em primeiro lugar como sujeito cognoscente possuidor de "modelos mentais" do "mundo exterior" que são transformados durante o processo informacional" (CAPURRO, 2003, p.8). Nesse momento, são inseridas teorias sobre os modelos mentais existentes no sujeito cognoscente, e que pertencem à estrutura cerebral de todo ser humano.

O terceiro paradigma é o social, uma vez que considera a informação inserida nas relações sociais estabelecidas entre os grupos sociais e a sociedade moderna. Nesse contexto, "o objeto da ciência da informação é o estudo das relações entre os discursos, áreas de conhecimento e documentos em relação às possíveis perspectivas ou pontos de acesso de distintas comunidades de usuários" (HJØRLAND apud CAPURRO, 2003, p.10). 
Nesse contexto, importa observar que as tecnologias digitais estão re(modelando) as formas de pensar a informação e o seu uso. A raiz da computação digital possui um "caráter tecnológico recente e se refere ao impacto da computação nos processos de produção, coleta, organização, interpretação, armazenagem, recuperação, disseminação, transformação e uso da informação [...]" (CAPURRO, 2003, p.6). Em decorrência, a inserção do estudo da computação digital no contexto da Ciência da Informação é conseqüência do estabelecimento, de acordo com Castells (2005) da Sociedade Informacional (c.f. CASTELLS, 2005), visto ser uma sociedade baseada no uso das tecnologias da informação e comunicação para seu desenvolvimento social.

De acordo com o panorama sucintamente apresentado, a Arquivologia, uma das disciplinas que compõem a Ciência da Informação, tem participado efetivamente dessa trajetória.

Em outros termos, a Arquivologia, ou Arquivística, disciplina que "[...] tem por objetivo o conhecimento da natureza dos arquivos e das teorias, métodos e técnicas a serem observados na sua constituição, organização, desenvolvimento e utilização" (CAMARGO; BELLOTTO, (1996, p.5), possibilita à seus profissionais, participar da gestão de documentos em diversos contextos, variados suportes visando as diferentes necessidades informacionais por parte de seus usuários.

Como todas as disciplinas do conhecimento, a Arquivologia é composta por fundamentos teóricos e metodológicos próprios e característicos da Área. Dentre eles destacam-se as funções arquivísticas voltadas para a gestão de documentos, assim denominadas uma vez que estão relacionadas ao conjunto de princípios e operações que dizem respeito à organização e tratamento dos arquivos. De acordo com as clássicas premissas apresentadas por Rousseau e Couture (1998, p.265), são em número de sete as funções dos arquivos: criação, avaliação, aquisição, classificação, conservação, descrição e difusão.

A criação ou produção refere-se a geração de documentos. Na produção de documentos, a constituição de um arquivo dá-se como conseqüência natural do exercício da atividade própria da instituição a que ele pertence. $A$ avaliação, realizada de acordo com critérios preestabelecidos, define o status do documento, sua destinação. A aquisição, "contempla a entrada de documentos nos arquivos [...]; refere-se ao arquivamento corrente e aos procedimentos de transferência e recolhimento de acervo" 
(SANTOS; INNARELLI; SOUSA, 2007, p.179). A classificação, diz respeito a criação e utilização de padrões que orientam a organização dos documentos de arquivos. A conservação abrange ações de preservação da integridade dos documentos, bem como as tecnologias. A descrição compreende o "conjunto de procedimentos que, a partir de elementos formais e de conteúdo, permitem a identificação de documentos e a elaboração de instrumentos de pesquisa" (CAMARGO; BELLOTTO, 1996, p.23, grifo das autoras). Considerada função básica dos organismos arquivísticos, a difusão está relacionada à disponibilização dos documentos, ao acesso para consultas.

Diante do exposto, torna-se evidente que a Arquivologia, por meio de seus fundamentos está habilitada a participar de forma efetiva na gestão de documentos. Ao preocupar-se com a criação, tratamento e divulgação das informações nos mais diversos contextos, a Área estabelece estreitas e competentes relações com o prontuário do paciente, aqui caracterizado como o documento arquivístico fundamental à saúde da população.

\section{PRONTUÁRIO DO PACIENTE}

Termo originário do latim promptuarim, de acordo com Ferreira (2004), o prontuário pode ser definido como "Lugar onde se guardam ou depositam as coisas de que se pode necessitar a qualquer instante; Manual de indicações úteis e Ficha (médica, policial, etc.) com os dados referentes a uma pessoa". Para o Conselho Federal de Medicina-CFM (BRASIL, 2002) o prontuário é caracterizado como

[...] conjunto de informações, sinais e imagens registradas, geradas a partir de fatos, acontecimentos e situações sobre a saúde do paciente e a assistência a ele prestada, de caráter legal, sigiloso e científico, utilizado para possibilitar a comunicação entre membros da equipe multiprofissional e a continuidade da assistência prestada ao indivíduo.

Dentro desse contexto, cabe ressaltar que os prontuários de uma instituição voltada à área da saúde constituem-se em documentos de extrema relevância, não somente no que diz respeito ao diagnóstico e tratamento prescrito nas enfermidades de cada paciente que é atendido, como também são considerados importantes fontes de informação a respeito de determinada patologia, seu histórico, desenvolvimento, prescrições, cura ou medidas paliativas, entre outras especificidades que a caracterizam. 
Sob outro enfoque, é possível afirmar que a importância dos prontuários aumenta de forma substancial se as instituições, além dos tratamentos realizados, dedicarem-se também ao uso e compartilhamento das informações geradas nos seus prontuários, como fonte de estudo, de pesquisa.

Corroborando a afirmação anterior, Marin; Massad; e Azevedo Neto (2003, p. 1) argumentam que

As informações registradas no prontuário médico [do paciente] vão subsidiar a continuidade e a verificação do estado evolutivo dos cuidados de saúde, quais procedimentos resultam em melhoria ou não do problema que originou a busca pelo atendimento, a identificação de novos problemas de saúde e as condutas diagnósticas e terapêuticas associadas.

Dando prosseguimento ao raciocínio, os autores, na mesma obra e página, ressaltam a relevância desses documentos ao asseverarem "[...] que o sistema de saúde de um país, é estabelecido graças ao que se tem documentado em um prontuário, uma vez que dele são extraídas as informações sobre a saúde dos indivíduos que formam uma comunidade e uma nação".

O valor probatório desse dossiê também não pode ser ignorado, pois, como indica o Conselho Regional de Medicina do Estado de Santa Catarina (2000, p. 1), o prontuário "[...] tem sido a principal peça de defesa do médico nos casos de denúncias por mau atendimento com indícios de imperícia, imprudência ou negligência, ou seja, na presunção da existência de erro médico".

Independentemente do suporte no qual está registrado, segundo o CFM (BRASIL,2002), no prontuário devem constar os seguintes itens:

a) Identificação do paciente - nome completo, data de nascimento, sexo, nome da mãe, naturalidade (indicando o município e o estado de nascimento), endereço completo (nome da via pública, número, complemento, bairro/distrito, município, estado e CEP);

b) Anamnese, exame físico, exames complementares solicitados e seus respectivos resultados, hipóteses diagnósticas, diagnóstico definitivo e tratamento efetuado;

c) Evolução diária do paciente, com data e hora, discriminação de todos os procedimentos aos quais foi submetido e identificação dos profissionais que as 
realizaram, assinados eletronicamente quando elaborados e/ou armazenados em meio eletrônico;

d) Nos prontuários em suporte de papel é obrigatória a legibilidade da letra do profissional que atendeu o paciente, bem como a identificação dos profissionais prestadores do atendimento. São também imprescindíveis a assinatura e o respectivo número do $\mathrm{CRM}$;

e) Nos casos emergenciais nos quais seja impossível a coleta da história clinica do paciente, deverá constar relato médico completo de todos os procedimentos realizados e que tenham possibilitado o diagnóstico e/ou a remoção para outra unidade.

Considerando as características, o teor das informações registradas, torna-se evidente o valor da documentação contida no prontuário para o paciente, para a equipe que presta atendimento à saúde e para a instituição.

Com relação ao paciente, os dados inscritos possibilitam atendimento, diagnóstico e tratamento de forma eficiente e eficaz. É para o paciente, também, um instrumento de defesa "no caso de possíveis prejuízos, e irregularidades ou reivindicações de direitos perante o médico, a equipe de saúde, o hospital e os poderes públicos." (POSSARI, 2008, p.23)

Para o médico, torna-se vital à sua práxis, já que um prontuário bem elaborado é um instrumento de grande valor para o tratamento da doença, pois fornece todos os dados já anotados, e serve ainda como instrumento de defesa em sua atividade profissional.

No que tange à equipe de saúde, - formada por profissionais que mantém vários tipos de contato com o paciente -, o prontuário possibilita, entre outros fatores, maior e melhor comunicação/integração entre os membros dessa equipe, o que resulta no aumento da qualidade do atendimento prestado.

Para o hospital que possui prontuários mais completos e bem elaborados, possibilita maior rotatividade de pacientes, reduz consideravelmente o uso indevido e repetido de serviços e equipamentos, otimizando os recursos disponíveis. O prontuário é um documento importantíssimo para sua defesa contra acusações e complicações de ordem técnica, ética ou jurídica que eventualmente possam ocorrer uma vez que possibilita o fornecimento, a qualquer tempo, de informações a respeito do tratamento realizado e do 
resultado alcançado até aquele momento. É também para a instituição, um demonstrativo fidedigno do padrão da qualidade do atendimento prestado (POSSARI, 2008).

No que diz respeito ao ensino e pesquisa, o prontuário é o instrumento que possibilita o conhecimento de inúmeros e diferentes casos com todas as variáveis antecedentes simultâneas e conseqüentes da doença. Permite o estudo do diagnóstico, a avaliação terapêutica. É campo para pesquisa e fonte de dados estatísticos acerca de determinada patologia, sua incidência, prevalência e mortalidade. Torna possível a verificação e comparação dos tratamentos e permite estabelecer uma análise da sua eficiência ou ineficiência (SERVIÇO..., 2002).

De um modo sintético, Van Ginneken e Moorman (apud COSTA, 2001), destacam o valor do prontuário como suporte à assistência ao paciente; como fonte para a avaliação e tomada de decisão e fonte de informação a ser compartilhada entre profissionais de saúde; suporte à pesquisa (pesquisa clínica, estudos epidemiológicos, avaliação da qualidade do atendimento e ensaios clínicos, entre outras); gerenciamento e serviços: (faturamento, autorização de procedimentos, administração, custos, etc.) e como documento legal dos atos médicos.

Considerando as contribuições acima mencionadas, torna-se óbvio que as informações contidas nos prontuários devem ser sistematicamente organizadas e tratadas, uma vez que, além de registrar as queixas do paciente, armazena informações a respeito dos cuidados prestados e dos custos decorrentes desses atendimentos.

Novaes (2003) salienta que os relatos apresentados pelos pacientes devem ser registrados em todos os encontros com os médicos, pois a enfermidade é um processo, e como tal, sofre mudanças que precisam estar registradas para que, após uma observação sistemática e minuciosa, possa ser diagnosticada e tratada. Ao destacar as informações contidas nos prontuários como fonte de pesquisa e estudo, percebe-se que nem sempre é fácil conseguir uma informação objetiva acerca da evolução do paciente, principalmente, se este possuir vários problemas de saúde e necessitar de vários profissionais da área, visto que esses mesmos profissionais geram individualmente, no prontuário, informações variadas. Importa mencionar que esses dados clínicos são, por sua vez, diversificados e inscritos em vários suportes, como as imagens geradas na radiologia, ultra-sonografia, tomografia computadorizada, nos gráficos, planilhas, e nas observações clínicas. Lawrence Weed (apud MARIN; MASSAD; AZEVEDO NETO, 2003, p. 4) preocupado com 
essa diversidade de informações, sugeriu a elaboração de registros de informações voltadas aos problemas do paciente, ou orientados para problemas, cujos objetivos eram os de identificar todos os problemas que iriam orientar as condutas adotadas pelos profissionais da saúde.

onde se identificam os problemas de saúde do paciente e as anotações registradas e seguidos de acordo com uma estrutura sistemática de registro de dados denominada SOAP pelo seu acrônimo em inglês $(S=$ queixas; $O=$ achados; $A=$ testes $e$ conclusões; $\mathrm{P}=$ plano de cuidado).

As informações que compõem os Prontuários do Paciente, durante muito tempo foram registradas em suporte físico, ou seja, o papel. Atualmente, com o advento da tecnologia, mais especificamente as aplicações de informática na área da saúde, tornouse possível que os prontuários em papel sejam migrados - por meio da digitalização - para o suporte eletrônico, como também que os Prontuários Eletrônicos dos Pacientes (PEP) ${ }^{2}$ já sejam criados nesse meio.

\subsection{Prontuário eletrônico do paciente}

Os primeiros sistemas de informação hospitalar informatizados surgiram na década de 60, cujo objetivo era a comunicação interna nos diferentes setores do hospital. Posteriormente esses sistemas passaram a armazenar alguns dados do prontuário. Em 1972, no Congresso patrocinado pelo National Center for Health Services Researchand Development e pelo National Center for Health Statistics dos Estados Unidos iniciou-se o processo para se estabelecer uma estrutura para os registros médicos ambulatoriais 0 que logo após propiciou o surgimento dos primeiros sistemas PEP.

A aprovação da normatização do prontuário eletrônico no Brasil deu-se por intermédio das Resoluções 1638 e 1639 do CFM. Com o uso da certificação digital, o prontuário eletrônico do paciente passa a ter presunção de validade jurídica. Em 2004, foi lançado pela Sociedade Brasileira de Informática em Saúde (SBIS) e o Conselho Federal de Medicina o Manual de Certificação para Sistemas de Registro Eletrônico em Saúde, no qual são estabelecidos critérios para que um prontuário eletrônico seja considerado seguro e com validade legal.

\footnotetext{
${ }^{2}$ Prontuário Eletrônico do Paciente (PEP); Registro Eletrônico do Paciente (REP); Registro Eletrônico de Saúde (RES), entre outros, são assim denominados os prontuários em suporte eletrônico.
} 
Segundo o Institute of Medicine (IOM) O PEP é um sistema de informação desenvolvido especificamente para atender ao usuário fornecendo dados completos e corretos, lembretes, sistemas de apoio às decisões, links para bases de conhecimento médico e outros recursos. Sob essa perspectiva, Marin (2003, p. 75) afirma que "a proposta básica do PEP é unir todos os diferentes tipos de dados produzidos em variados formatos, em épocas diferentes, feitos por diferentes profissionais da equipe de saúde em distintos locais".

Sabatini (2002) apud Pinto (2006, p.37) argumenta que, com chegada dos dispositivos eletrônicos, o Prontuário do Paciente, caracterizado como um documento passivo, muitas vezes difícil de ser entendido e distante do universo semântico do paciente, passou a ser percebido como "um instrumento ativo, uma central de serviços de informação, um promotor de saúde e de prevenção de problemas, e um educador de pacientes e divulgador de informações confiáveis sobre medicina e saúde".

Pinto, na mesma obra, reitera a citação anterior ao ressaltar, baseada em Gremy (1987), que o prontuário do paciente, "que antes tinha função de documentar as informações da saúde e da doença do paciente, hoje se tornou complexo, passando a ter um papel de fundamental importância na sociedade contemporânea", uma vez que, segundo a autora, o prontuário propicia o compartilhamento de informação e conhecimento entre diversos profissionais e suas especialidades; é o documento de base legal para as ações médicas e dos pacientes; é uma fonte de pesquisa clínica e atualização contínua, tratamentos e cuidados praticados nos pacientes; apresentam e documentam custos e pagamentos, base para toda gestão hospitalar.

Entretanto, vale ressaltar, o PEP, como sistema informatizado, demanda ações voltadas à segurança, proteção e preservação dos dados nele registrados. A segurança desses dados é de extrema importância para se manter a fidedignidade das informações. Para isso são utilizados procedimentos que autorizam o acesso somente a quem é permitido.

Como todo e qualquer sistema de informação, o prontuário, possui características positivas e negativas. Baseadas em Pinto (2006), são estabelecidas comparações entre o Prontuário Eletrônico do Paciente e o Prontuário "tradicional", como se segue.

Com relação ao Prontuário Eletrônico do Paciente, os pontos positivos são: 
redução no tempo de atendimento e os custos decorrentes; possibilidades de reconstrução histórica e completa dos casos atendidos, tratamentos prescritos etc.; acesso simultâneo em locais distintos; legibilidade nas informações; contribuição para a pesquisa científica; integralidade dos variados tipos de dados/informações; facilidade na organização e no acesso às informações; otimização do espaço de arquivamento. Em contrapartida, o PEP, demanda grande investimento em hardware, software e treinamento; pode suscitar, por parte daqueles que o utilizam, resistência às mudanças; ocasiona demora na sua implantação, como todo e qualquer sistema informatizado; requer a manutenção dos prontuários de papel para fins jurídicos, quando não há certificação do processo; possibilidade de falhas na tecnologia.

Com o prontuário do paciente em formato "tradicional", há maior liberdade na maneira de escrever; ele é facilmente transportado; de fácil no manuseio, não requerendo treinamento para tanto; nunca fica "fora do ar". Com relação aos pontos negativos, o prontuário em papel, muitas vezes pode dificultar a compreensão de seu conteúdo devido a fatores como: ilegibilidade, falta de padronização na ordenação das informações, inexistência de controle terminológico entre outros; impossibilita o acesso por vários profissionais ao mesmo tempo; pode ser extraviado; fragilidade do suporte.

Observa-se pelo descrito acima que as vantagens do PEP em relação ao Prontuário de Papel são comprovadamente mais relevantes, destacando-se a maior integralidade dos dados, a interoperabilidade dos sistemas; fatores que constituem o atributo essencial na qualidade dos cuidados ao paciente, dos serviços prestados no sistema de saúde de um país. Outra vantagem considerável também é a diminuição do volume documental e conseqüentemente, do espaço físico que o prontuário de papel demanda.

Todavia, como mencionado na literatura, a implantação do PEP requer, além de alto investimento em equipamentos, capacitação de pessoal, pois possui uma funcionalidade mais complexa, além de demandar manutenção periódica e cuidados na preservação das informações constantes nesse repositório digital. Nesse cenário, importa salientar que, mais do que uma transposição do papel para o meio eletrônico, o "novo" formato do prontuário exige, quer seja por parte dos profissionais da Saúde, quer seja do setor que administra a instituição, mudanças expressivas em suas rotinas. Em virtude dessas questões, - ainda que superficialmente abordadas nesse estudo -, o PEP não pode ser considerado simplesmente como um produto, resultante das anotações dos profissionais 
que atenderam o cliente, ou 0 paciente. As informações geradas pelos diversos profissionais a partir dos cuidados prestados ao paciente dão origem a um fluxo de informações que deve ser constantemente alimentado e adequado as necessidades de seus usuários, caracterizando-o como um processo complexo e dinâmico.

\section{CONSIDERAÇÕES FINAIS}

Preservar os registros do conhecimento é essencial para a sobrevivência e desenvolvimento do ser humano. Nessa perspectiva, os prontuários gerados em instituições voltadas à área da saúde, constituem-se em documentos de extrema importância para pesquisa médica, administrativa, jurídica e acadêmica. Além disso, o prontuário, no formato eletrônico, proporciona mais rapidez na localização da informação, e consequentemente maior presteza no atendimento do paciente.

Bertolli Filho (2006, p. 15) evidencia a relevância dos prontuários ao afirmar que "[...] no rol de documentos constitutivos dos arquivos médicos, afloram como peças preciosas que possibilitam fixar cenários, confirmar saberes e desvelar sensibilidade, enfim, viabilizar novas possibilidades de apreensão da memória da Saúde [...]”.

Dentro desse cenário, portanto, torna-se consensual que o registro sistematizado das informações contidas nos prontuários são elementos importantes para o atendimento do paciente uma vez que possibilita a implantação de medidas que proporcionem ações de prevenção na área da saúde. Sob outro enfoque, permite ainda, o resgate da história da instituição e dos tratamentos empregados. Serve, também, como suporte à gestão de serviços no âmbito da saúde e como valiosa fonte de informações para geração de conhecimento, pesquisa e estudo. Nesse sentido, os prontuários, sejam eles em formato impresso ou eletrônico, devem ser elaborados, organizados e tratados adequadamente, pois, transformações sociais e desenvolvimento econômico e científico decorrentes de tomadas de decisões, dependem crucialmente das informações que os compõem.

A apresentação - ainda que não exaustiva - das ações acima descritas, não deixam dúvidas quanto à importância do Arquivista e dos fundamentos que norteiam suas atividades, na implantação e utilização do prontuário em unidades de saúde. Tal premissa, entretanto, não é nova ou original. Ainda que seja inexpressivo o número de profissionais trabalhando em parceria com as organizações voltadas à saúde, vários 
estudiosos do assunto e entre eles, Pinto (2006, p 46) ao referir-se especificamente ao dossiê em formato eletrônico, concluiu que

Finalmente, entendemos que o PEP é um documento técnico suis generis e, como qualquer outro, precisa de tratamento adequado a fim de que as informações nele contidas possam ser recuperadas de maneira eficaz e eficiente. Portanto, é um tipo de documento que precisa ser explorado no contexto da Ciência da Informação.

À guiza de encerramento, importa mencionar que, apesar das instituições de saúde já estarem se preparando para utilizar ou mesmo utilizando o PEP, se não houver um comprometimento efetivo por parte de todos os seus setores e profissionais com relação ao preenchimento desse dossiê, respeitando princípios já consagrados pela Arquivologia, em especial àqueles que perpassam as atividades de tratamento documental, serão insignificantes os benefícios oriundos desse novo processo de registro a respeito da saúde de uma população.

\section{REFERÊNCIAS}

ARAUJO, R. N. U. A Informação como determinante de sucesso para micro e pequenas empresas. In: TARGINO, M. G.; CASTRO, M. M. M. R. N. (Orgs.). Desafiando os domínios da informação. Terezina: EDUFPI, 2002, p. 229-262.

BERTOLLI FILHO, C. Prontuários médicos e a memória da saúde brasileira. BIS, Memória e História da Saúde em São Paulo, São Pulo, n. 38, p. 12-15, abr. 2006.

BRASIL. CONSELHO FEDERAL DE MEDICINA. Resolução n. 1638 de 10 de julho de 2002. Diário Oficial, Brasília, 10 jul. 2002. Seção 1, p. 124-5.

CAMARGO, A. M. de A.; BELLOTTO, H.L. (Cols.). Dicionário de terminologia arquivística. São Paulo: Secretaria de Estado e Cultura, 1996.

CAPURRO, R. Epistemologia e ciência da Informação. IN: ENCONTRO NACIONAL DE PESQUISA EM CIÉNCIA DA INFORMAÇÃO, 5., 2003, Belo Horizonte. Anais Eletrônicos...

CASTELLS, M. A era da informação: economia, sociedade e cultura: a sociedade em rede: I volume. 8.ed. São Paulo: Paz e Terra, 2005. 698p.

CHOO, Chun Wei. Gestão da informação para a organização inteligente: a arte de explorar o meio ambiente. Lisboa: Caminho, 2003.

CONSELHO REGIONAL DE MEDICINA DO ESTADO DE SANTA CATARINA. Manual de orientação ética e disciplinar. 2 ed. rev. Atual. Florianópolis, 2000. Disponível em:

<http://www.portalmedico.org.br/regional/crmsc/manual/parte3b.htm>. Acesso em: 2 dez. 2007. 
COSTA, C. G. A. da. Desenvolvimento e avaliação tecnológica de um sistema de prontuário eletrônico do paciente, baseado nos paradigmas da world wide web e da engenharia de software. 2001. 268f. Dissertação (Mestrado em Engenharia Elétrica). Faculdade de Engenharia Elétrica e de Computação da Universidade Estadual de Campinas, Campinas, 2001.

DAHLBERG, I. Knowledge organization: its scope and possibilities. Knowlegde Organization, Wurzburg, v. 20, n. 4, p. 211-22, 1993.

DAVENPORT, T. H. Ecologia da informação: por que só a tecnologia não basta para o sucesso na era da informação. São Paulo: Futura, 2000.

FERREIRA, A. B. de H. Novo dicionário eletrônico Aurélio. Versão 5.0. [S.I.]: Editora Positivo, 2004. CD ROM.

INSTITUTE OF MEDICINE. The computer-based patient record: an essencial technology of health care. Washington, D.C: Division of Health Care Services, 1997.

MARIN, H. de F. Os componentes de Enfermagem do Prontuário Eletrônico do Paciente. In: MASSAD, E.; MARIN, H. de F.; AZEVEDO NETO, R. S. de. (Eds.). O prontuário eletrônico do paciente na assistência, informação e conhecimento médico. São Paulo: Ed. do Autor, 2003. p.73-84.

MARIN, H.de F.; MASSAD, E.; AZEVEDO NETO, R. S. de. Prontuário eletrônico do paciente: definições e conceitos. In: MASSAD, E.; MARIN, H. de F.; AZEVEDO NETO, R. S. de. (Eds.). O prontuário eletrônico do paciente na assistência, informação e conhecimento médico. São Paulo: Ed. do Autor, 2003. p.1-20.

MC INERNEY, C. R. Compartilhamento e gestão do conhecimento: profissionais da informação em um ambiente de confiança mútua. In: e conhecimento. Brasília: IBICT, UNESCO, 2006. . Inteligência, informação

MEDEIROS, F. G. S. Sistema de Informação em saúde: considerações fundamentais. In: TARGINO, M. G.; CASTRO, M. M. M. R. N. (Orgs.). Desafiando os domínios da informação. Terezina: EDUFPI, 2002, p. 119-127.

MOTA, F.R.L. O prontuário eletrônico do paciente e o processo de competência informacional. Enc. Bibli: R.Eletr. Bibliotecon. Cl. Inf., Florianópolis, v.11, n.22, p.53-70, 2006.

MOTA, F.R.L.; BABÊTTO, H.S. Processamento e compartilhamento da informação em prontuários eletrônicos. Disponível em: < www.sbis.org.br/cbis9/arquivos/254.trf. >. Acesso em: 02 de mar. de 2007.

NONAKA, I.; TAKEUCHI, H. Criação do conhecimento na empresa: como as empresas japonesas geram a dinâmica da informação. Rio de Janeiro: Campos, 1997.

NOVAES, H. M. D. Diagnosticar e classificar: o limite do olhar. São Paulo: Tese (Doutorado em Medicina). Faculdade de Medicina da USP, 1987. 
POSSARI, J.F. Prontuário do paciente e os registros de enfermagem. 2.ed. São Paulo: látria, 2008.

PINTO, V. B. Prontuário eletrônico do paciente: documento técnico de informação e comunicação do domínio da saúde. Enc. Bibli: R.Eletr. Bibliotecon. Cl. Inf., Florianópolis: v.11, n.21, p. 34-48, 2006.

PROBST, G. Gestão do conhecimento: os elementos construtivos do sucesso. Porto Alegre: Bookman, 2006.

ROUSSEAU, J-Y.; COUTURE, C. Os fundamentos da disciplina arquivística. Lisboa: Publicações Dom Quixote, 1998.

SANTOS, V. B, dos; INNARELLI, H. C.; SOUSA, R. T. B de (Orgs.). Arquivística: temas contemporâneos: classificação, preservação digital, gestão do conhecimento. Brasília: SENAC, 2007.

SERVIÇO de Prontuário do Paciente. Goiânia, 2002. Disponível em: <http://www.aguavi va.mus.br/enfermateca/Trabalhos/ServicoProntuarioPaciente.htm>. Acesso em: 18 ago. 2007.

SOUZA, F. C. Organização do conhecimento na sociedade. Florianópolis: UFSC/CED, Núcleo de Publicações, 1998 (Cadernos CED, 1).

WILSON, I. D. A problemática da gestão do conhecimento. In: TARAPANOFF, Kira (Org.). Inteligência, informação e conhecimento. Brasília: IBICT, UNESCO, 2006, p.37-55.

\section{Title}

The patient's medical record and the assumptions archivist: a close and useful dialogue.

\section{Abstract}

Considered as a multifunctional document for the Health area, the Patient's Medical Record is dealt with in its electronic and paper versions. As for the difficulties found in the implantation and use of the Patient's Electronic Record, the confrontation between the two formats mentioned above, demonstrates the priority of the Electronic Record and distinguishes it as a predominant element concerning the improvement of health systems and services of a country. Taking its specificities into account, it is emphasized, among other factors, the role of the assumptions archivist in the organization of the information as well as of the knowledge registered in these medical records.

\section{Keywords}

Knowledge Organization. Information Organization. Patient's Medical Record. Patient's Electronic Record (PER). Assumptions Archivist. 


\section{Título}

Registros de pacientes y los supuestos archivero: los diálogos estrecha y fructífera

\section{Resumen}

Considerado el documento multifuncional para el área de Salud, el historial del paciente y discutido en su papel y medios electrónicos. Pese a las dificultades en el despliegue y uso de la electrónica de registro de pacientes, la comparación entre los dos formatos mencionados anteriormente, indica la primacía de los registros médicos electrónicos y caracterizado como un factor dominante a la hora de mejorar los sistemas y servicios de salud en un país. Teniendo en cuenta sus especiales características, los requisitos básicos para su pleno uso, entre otros factores, destaca el papel de los supuestos archivero en la organización de la información y conocimientos registrados en estos archivos.

\section{Palabras clave}

Organización del conocimiento. Organización de la información. Registro del paciente. Electronic Patient Record (EPR). Supuestos Archivero.

Recebido em: 20.03.2010

Aceito em: 15.09 .2010 